\title{
Effects of Saccharomyces Cerevisiae Fermentation Products on the Microbial Community throughout the Gastrointestinal Tract of Calves
}

\author{
Jianxin Xiao ${ }^{1}$, Gibson M. Alugongo ${ }^{1}{ }^{\oplus}$, Shoukun Ji ${ }^{1}$, Zhaohai $\mathrm{Wu}^{1}{ }^{1}$, Shuangzhao Dong ${ }^{1}$, \\ Shengi Li ${ }^{1}$, Ilkyu Yoon ${ }^{2}$, Ruby Chung ${ }^{2}$ and Zhijun Cao ${ }^{1, *}$ \\ 1 State Key Laboratory of Animal Nutrition, Beijing Engineering Technology Research Center of Raw Milk \\ Quality and Safety Control, College of Animal Science and Technology, China Agricultural University, \\ Beijing 100193, China; dairyxiao@gmail.com (J.X.); maswayi@yahoo.com (G.M.A.); jishoukun@163.com (S.J.); \\ wzh07128@163.com (Z.W.); zhaoddr@163.com (S.D.); lisheng0677@163.com (S.L.) \\ 2 Diamond V Inc., Cedar Rapids, IA 52404, USA; iyoon@diamondv.com (I.Y.); rchung@diamondv.com (R.C.) \\ * Correspondence: caozhijun@cau.edu.cn; Tel.: +86-10-62733746
}

Received: 15 October 2018; Accepted: 13 December 2018; Published: 21 December 2018

check for updates

Simple Summary: Saccharomyces cerevisiae fermentation products (SCFP) are widely used for dairy cows and have been suggested to improve calf performance and health. However, the changes in microbial community along the gut in calves supplemented with SCFP have not been investigated extensively. This manuscript exhibited that calves supplemented with Saccharomyces cerevisiae fermentation products changed the microbial community of GIT and stimulated fibrolytic bacteria (Lachnospiraceae and Ruminococcaceae) colonization in early rumen and large intestine, respectively. Those alternations of microbiota in GIT might explain how SCFP works in calves.

\begin{abstract}
The effect of Saccharomyces cerevisiae fermentation products (SCFP) on improving growth and health of calves could be attributed to the ability of SCFP to modulate the microbiota in the gastrointestinal tract (GIT). However, the changes in microbial community along the gut in calves supplemented with SCFP have not been investigated extensively. The aims of this study were to investigate the effect of SCFP on microbial communities in each sites of GIT using high-throughput sequencing technique. Fifteen Holstein male calves were used and randomly assigned to 1 of the 3 treatments including a calf starter containing 0 (Control, CON), 0.5 (SCFP1) or 1\% SCFP (SCFP2, Original XPC, Diamond V, Cedar Rapids, IA, USA) of dry matter from day 4 to 56 . The supplemented calves were fed with an additional $1 \mathrm{~g} / \mathrm{d}$ SCFP (SmartCare, Diamond V, Cedar Rapids, IA, USA) in milk from day 2 to 30 . Rumen fluid was sampled at day 28 of age via esophageal tube. All calves were slaughtered and gastrointestinal samples collected on day 56. Inclusion of SCFP increased the microbial species richness in the large intestine. The SCFP also affected the bacterial community at an early age in the rumen and later in rectum microbiota. Supplementation of SCFP stimulated colonization by fibrolytic bacteria (Lachnospiraceae and Ruminococcaceae) in rumen and large intestine, respectively. No differences were found between SCFP1 and SCFP2. This is the first study to analyze the effect of SCFP on bacterial community of the GIT microbiota in calves. The results provide the basic bacterial community information, which helps us understand the mechanism of action of SCFP for improving the health and performance of pre-weaning calf.
\end{abstract}

Keywords: calf; Saccharomyces cerevisiae fermentation product; bacterial community; species richness 


\section{Introduction}

The rumen bacteria are the most important microorganisms in the gastrointestinal tract (GIT), where they interact with the host, contributing to its performance and health [1]. Recent evidence shows that the colonization by microorganisms in rumen [2] and intestines [3,4] occurs immediately after birth, with some of the essential microbes involved in mature rumen function present as early as at day 1 [5]. Both liquid and solid feed are likely to influence the bacterial community in the fore [1] and hind gut $[6,7]$ of pre-weaning calves. As an important functional part of diets, feed additives, are also effective in altering the gut bacterial community and related animal health and performance [8,9].

Saccharomyces cerevisiae fermentation products (SCFP) are common feed additives in the dairy industry. The yeast fermentation process produces biologically active compounds including oligosaccharides, organic acids, amino acids and peptides [10] which can improve the survival of calves under stress, resulting in greater profit margins [11]. Other benefits of SCFP in calves include improved ADG and body structure [12], enhanced VFA production [13] and a more anatomically and physiologically developed rumen [14]. Furthermore, SCFP have the potential to influence the microbial composition in the GIT. In mature animals, SCFP stimulated the growth of ruminal fiber-digesting bacteria (Ruminococcus albus, Ruminococcus flavefaciens and Fibrobacter succinogenes) and lactate-utilizing bacteria [15-17]. Saccharomyces cerevisiae fermentation products can be supplemented in milk, milk replacer, and/or calf starter. Supplementing SCFP in milk or milk replacer can ensure the consumption of the product from very early in life of the calf. We have previously demonstrated that SCFP can influence Butyrivibrio and Prevotella in the rumen fluid [18]. These calves were fed Original XPC (XPC; Diamond V, Cedar Rapids, IA, USA) and SmartCare (SC; Diamond V, Cedar Rapids, IA, USA) which are SCFPs supplemented in starter and milk (or milk replacer), respectively. However, the effect of supplementing SCFP in diets of calves on hindgut microbial community has not yet been fully elucidated. Therefore, to further understand whether and how SCFP modulate the microbial population along the GIT in pre-weaning Holstein calves, we investigated microbial composition at different taxonomic levels and in different sites in the first 56 days of life.

\section{Materials and Methods}

\subsection{Ethics Approval}

The experimental design and procedures were executed according to the protocols approved by the Ethical Committee of the College of Animal Science and Technology, China Agricultural University (protocol number: 2013-5-LZ). Animal care and use strictly followed the Regulations for the Administration of Affairs Concerning Experimental Animals, National Committee of Science and Technology of China (14 November 1988) and Instructive Notions with Respect to Caring for Laboratory Animals, Ministry of Science and Technology of China (30 September 2006).

\subsection{Animal Trials}

The experiment was conducted using 15 Holstein male calves, which were separated from their dams immediately after birth. All calves averaged $41.8 \pm 2.5 \mathrm{~kg}$ BW and only calves with successful passive transfer of immunity were used $(\geq 5.5 \mathrm{~g} / \mathrm{dL}$ of serum total protein, determined by clinical refractometer $24 \mathrm{~h}$ after birth). The calves were raised in hutches with free access to calf starter and clean water from day 4. Straw was used as bedding and renewed weekly. All calves received $4 \mathrm{~L}$ of colostrum (Brix values $\geq 22 \%$ ) within $1 \mathrm{~h}$ of birth, and thereafter pasteurized milk were fed twice daily at 800 and 1,500 h from day 2 to day 56, calves received $6 \mathrm{~L} / \mathrm{d}$ of milk from day 2 to 10, $8 \mathrm{~L} / \mathrm{d}$ from day 11 to $42,6 \mathrm{~L} / \mathrm{d}$ from day 43 to 49 and $4 \mathrm{~L} / \mathrm{d}$ afterwards until weaning on day 56. Calves were randomly assigned to 1 of the 3 treatments including CON (no SCFP), SCFP1 $(1 \mathrm{~g} / \mathrm{d}$ SmartCare in the milk $+0.5 \%$ XPC in the starter), and SCFP2 ( $1 \mathrm{~g} / \mathrm{d}$ SmartCare in the milk + 1\% XPC in the starter). The XPC was incorporated in the texturized calf starter (19\% CP; HONNEUR Nutritional Technology Co., Ltd., Beijing, China) from day 4 to day 56 while SmartCare was added into milk daily in the morning milk feeding from day 2 to day 30. 
The calves were weaned and harvested on day 56. SmartCare is a water-soluble product that can be supplemented to milk and XPC is a dry feed product that can be added in starter. The combination of these products is the basis for Diamond V's dairy calf program during pre-weaning phase.

Ingredients and chemical composition of calf starter was identical among treatments with the exception of SCFP content, which was replaced as a part of corn germ meal. Nutrient composition of starter met or exceeded the requirements for pre-weaned Holstein calves [19], and was composed of steam flaked corn $(33.1 \%)$, wheat bran $(7.6 \%)$, canola meal $(8.0 \%)$, extruded soybean $(4.2 \%)$, soybean meal (14.3\%), corn germ meal $(8.5,8.0$ and $7.5 \%$ for CON, SCFP1 and SCFP2, respectively), rice powder $(4.2 \%)$, milk powder (0.4\%), DDGS (16.0\%), $\mathrm{CaCO}_{3}(1.4 \%), \mathrm{CaHPO}_{4}(1.0 \%), \mathrm{NaCl}(0.7 \%)$, mycotoxin binder $(0.1 \%)$, premix compound $(0.5 \%)$ and XPC $(0,0.5$ and $1 \%$ for CON, SCFP1 and SCFP2, respectively). Starter and water were offered ad libitum intake $1 \mathrm{~h}$ after milk feeding.

\subsection{Sample Collections}

\subsubsection{Rumen Fluid Collection on Day 28}

Rumen fluid was collected on day 28 of age by a flexible esophageal tube (6 $\mathrm{mm}$ of inner diameter and $2 \mathrm{~mm}$ of wall thickness; Anscitech Co., Ltd., Wuhan, Hubei, China) from all 15 calves (five per treatment) $4 \mathrm{~h}$ after the morning milk feeding. The first $10 \mathrm{~mL}$ of rumen fluid was discarded to avoid saliva contamination. Rumen liquid sample (RL28) was obtained by filtering rumen fluid through four layers of cheesecloth, and $10 \mathrm{~mL}$ of the liquid (RL28) was immediately frozen in liquid nitrogen and stored at $-80^{\circ} \mathrm{C}$ until DNA extraction.

\subsubsection{Collection of Rumen and Intestine Contents on Day 56}

All calves were slaughtered and samples of gastrointestinal contents were collected on day 56 of age. After slaughtering, the abdominal cavity was immediately opened and each region of digestive tract (rumen, duodenum, cecum, and rectum) was isolated and tied off. The rumen solid (RS56) and liquid (RL56) fractions were obtained by squeezing the rumen digest samples through four layers of sterile cheesecloth. Rumen solid (RS56), RL28, RL56, intestinal contents from duodenum (DC56), cecum (CC56) and rectum (RC56) were separately placed into sterile tubes, snap-frozen in liquid nitrogen, and then stored at $-80^{\circ} \mathrm{C}$ pending further analysis.

\subsection{DNA Isolation and Illumina Hiseq Sequencing}

Rumen and intestinal samples were sent to Beijing Computing Center (Beijing, China) for DNA extraction, $16 \mathrm{~S}$ rRNA gene amplification and sequencing. DNA was extracted from $2.5 \mathrm{~g}$ rumen solid fraction, $400 \mu \mathrm{L}$ rumen fluid fraction and duodenum contents, $0.5 \mathrm{~g}$ cecum contents and $200 \mathrm{mg}$ rectum contents using QIAamp DNA Mini Kit (Qiagen, Hilden, Germany) following the manufacturer's protocol.

For illumina Hiseq sequencing, the $\mathrm{V} 3$ region of the $16 \mathrm{~S}$ rRNA genes was amplified using primers 343F (5'-GATCCTACGGGAGGCAGCA-3') and 534R (5'-GCTTACCGCGGCTGCTGGC- $\left.3^{\prime}\right)$ with barcodes. The NEB Next Ultra DNA Sample Prep Kit (NEB, Ipswich, MA, USA) was used to generate sequencing libraries as per manufacturer's instructions and standard Illumina sample-preparation protocol [20]. The Agilent Bioanalyzer 2100 system (Agilent Technologies, Palo Alto, CA, USA) and Qubit 2.0 Fluorometer (Life technologies, Grand Island, NY, USA) were used to evaluate the library quality and subsequently sequenced on an Illumina Hiseq 2500 platform. Paired-end reads with $250-300$ bp were generated.

\subsection{Data Processing and Analysis}

\subsubsection{Quality Control and Paired-End Reads Assemblies}

Quality sequences with a score of $>30$ were obtained by FastQC (Version 0.11.3, Babraham Bioinformatics, Babraham, UK). In order to obtain intact amplicons, paired-end reads with no 
mismatches from the original DNA fragments were merged using FLASH (Version 1.2.7, Adobe, San Jose, CA, USA) [21]. Concatenated and chimeric sequences were detected and subsequently filtered out by USEARCH (Version 6.1, Robert Edgar, Tiburon, CA, USA). Trimmed sequences were uploaded to QIIME (Version 1.8.0, Gregory Caporaso, Boulder, CO, USA) for further analysis.

\subsubsection{OTU Cluster and Species Annotation}

Sequence analysis was conducted using QIIME pipeline (Version 1.8.0, Gregory Caporaso, Boulder, CO, USA) [22]. Trimmed sequences were assigned to different samples based on barcodes and binned into operational taxonomic units (OTUs) by clustering sequences with a $97 \%$ similarity using the UCLUST software (Version 1.2.22, Robert Edgar, Tiburon, CA, USA) after removal of barcode and primers. The Greengene database and RDP classifier were used to classify the generated OTUs [23,24]. To reduce systematic variation and ensure the compatibility of the species diversity between the samples [25], the threshold of standardized sequences was set at 70,000 sequences (corresponding to the number of sequences in the minimum data set).

\subsubsection{Data Analysis}

Alpha diversity indices (ACE, Chao1, Shannon and Simpson) were determined using QIIME pipeline (Version 1.8.0) [22]. The beta diversity indices, principal coordinate analysis (PCoA) and ANOSIM analysis between samples were determined based on Bray-Curtis metrics with Vegan package in R (Version 2.4-1, Microsoft, Redmond, WA, USA) [26]. Heatmaps were produced by heatmap packages exhibiting differences in major bacterial community OTUs (top 80 OTUs) among dietary groups at different sampling sites (RL28, RL56, RS56, DC56, CC56 and RC56). Venn diagrams were created to characterize the overlap of OTUs present among dietary groups within each sampling site using the gplots package in R [27]. Singleton OTUs were removed from this analysis. Microbial composition graphs and bacterial abundance were subsequently generated under different classification levels. One rumen liquid sample, from CON and SCFP2 each collected at day 28 (RL28) was excluded from all the above analysis because they contained an unusually low number of sequences and huge differences within group.

Treatment effects were assessed using all 15 calves (88 samples). Variables of alpha diversity indices and bacterial abundance were analyzed separately by each sampling site using GLM procedure of SAS 9.2 (SAS Inst. Inc., Cary, NC, USA) with the fixed effect of treatment and the random effect of calf nested within treatment. Significant differences were indicated at $p$ value $\leq 0.05$. Comparison between treatments was carried out in SAS 9.2 (SAS Inst. Inc., Cary, NC, USA) using contrast statement to test (1) CON vs. SCFP (SCFP1 and SCFP2), and (2) SCFP1 vs. SCFP2. The Bonferroni correction was used when comparing the taxa abundance between treatments.

\subsection{Nucleotide Sequence Accession Numbers}

The identified sequences were deposited in the database at NCBI under SRX1744697, SRX1744731, SRX1744733, SRX1744734, SRX1744735 and SRX1744736.

\section{Results}

\subsection{Sequences and OTUs}

For each individual (15 male calves), the samples included RL28, RL56, RS56, DC56, CC56 and RC56. After sequencing and sequence trimming, a total number of $14,556,361$ quality reads were obtained from three treatments (CON, 4,926,750; SCFP1, 4,802,409; SCFP2, 4,827,202). The average reads per sample were 161,737. Among the 88 samples, an average of 1582, 1563 and 1588 OTUs were detected for CON, SCFP1 and SCFP2, respectively. The average and range of sequences and OTUs of each treatment in different sites were presented in Table 1. Good's coverage was high with an average of 0.99 across all samples, which imply sufficient depth in sequencing in the present study. 
Table 1. Diversity estimation of the $16 \mathrm{~S}$ rRNA gene libraries of all 88 samples.

\begin{tabular}{|c|c|c|c|}
\hline Sites/Groups ${ }^{\text {a }}$ & $\mathbf{N}^{\mathbf{b}}$ & Average Reads & Average OTUs ${ }^{c}$ \\
\hline \multicolumn{4}{|l|}{ RL28 } \\
\hline $\mathrm{CON}$ & 4 & 160,855 & 1101 \\
\hline SCFP1 & 5 & 155,655 & 1042 \\
\hline SCFP2 & 4 & 133,215 & 891 \\
\hline \multicolumn{4}{|l|}{ RL56 } \\
\hline $\mathrm{CON}$ & 5 & 158,254 & 1059 \\
\hline SCFP1 & 5 & 177,990 & 928 \\
\hline SCFP2 & 5 & 161,029 & 962 \\
\hline \multicolumn{4}{|l|}{ RS56 } \\
\hline $\mathrm{CON}$ & 5 & 144,101 & 1120 \\
\hline SCFP1 & 5 & 119,776 & 1075 \\
\hline SCFP2 & 5 & 141,623 & 1153 \\
\hline \multicolumn{4}{|l|}{ DC56 } \\
\hline $\mathrm{CON}$ & 5 & 156,828 & 1877 \\
\hline SCFP1 & 5 & 118,575 & 1045 \\
\hline SCFP2 & 5 & 161,956 & 1053 \\
\hline \multicolumn{4}{|l|}{ CC56 } \\
\hline $\mathrm{CON}$ & 5 & 183,365 & 2084 \\
\hline SCFP1 & 5 & 185,415 & 2460 \\
\hline SCFP2 & 5 & 178,122 & 2561 \\
\hline \multicolumn{4}{|l|}{ RC56 } \\
\hline $\mathrm{CON}$ & 5 & 184,172 & 2338 \\
\hline SCFP1 & 5 & 203,071 & 2829 \\
\hline SCFP2 & 5 & 196,179 & 2827 \\
\hline
\end{tabular}

${ }^{\text {a }}$ RL28 = Rumen liquid portions at day 28, RL56 = Rumen liquid portions at day 56, RS56 = Rumen solid contents at day 56, DC56 = Duodenal contents at day 56, CC56 = Cecal contents at day 56, RC56 = Rectal contents at day 56, RN56 = Rumen contents without fluid at day $56 . \mathrm{CON}=$ No SmartCare and XPC; SCFP $1=1 \mathrm{~g} / \mathrm{hd} / \mathrm{d}$ SmartCare + $0.5 \%$ XPC; SCFP2 $=1 \mathrm{~g} / / \mathrm{hd} / \mathrm{d}$ SmartCare $+1 \%$ XPC. ${ }^{\mathrm{b}} \mathrm{N}=$ the number of calves was used. ${ }^{\mathrm{c}}$ OTUs = Operational Taxonomic Units.

\subsection{Impact of SCFP on Microbiota}

\subsubsection{Microbial Richness and Diversity}

Microbial species richness and diversity indices (ACE, Chao1, Shannon, Simpson) were different between sites, with higher abundance in large intestine compared to the rumen (Table 2). We found out that, SCFP can influence microbial richness and diversity in rumen, duodenum and large intestine in different ways (Figure 1a-d). ACE and OTU numbers, as indices of microbial richness, decreased in rumen (RL56, $p=0.09$ and $p=0.11$ ) and duodenum (DC56, $p=0.06$ and $p=0.04$ ), but increased in the large intestine (CC56, $p=0.04$ and $p=0.04 ; \mathrm{RC} 56, p=0.01$ and $p=0.02$ ) when SCFP was supplemented in the diet. Microbial diversity (evenness), Shannon $(p=0.08)$ and Simpson $(p=0.10)$ tended to decrease only in RL28. Higher level of SCFP in the diet did not exhibit further influence under the conditions of this trial.

Table 2. The effect of gastrointestinal sites on the estimates of microbial richness and diversity in calves.

\begin{tabular}{cccccccc}
\hline \multirow{2}{*}{ Item/Index $^{1}$} & $\mathbf{N}^{2}$ & RL56 & RS56 & DC56 & CC56 & RC56 & SEM \\
\cline { 3 - 7 } & & \multicolumn{7}{c}{ Mean $^{3}$} \\
\hline ACE & 15 & $2038.2^{\mathrm{a}}$ & $2360.9^{\mathrm{ab}}$ & $2685.3^{\mathrm{b}}$ & $4358.6^{\mathrm{c}}$ & $4893.5^{\mathrm{c}}$ & 156.6 \\
Chao1 & 15 & $1998.9^{\mathrm{a}}$ & $2293.6^{\mathrm{a}}$ & $2537.7^{\mathrm{a}}$ & $4271.7^{\mathrm{b}}$ & $4810.1^{\mathrm{b}}$ & 153.4 \\
Shannon & 15 & $4.88^{\mathrm{a}}$ & $5.33^{\mathrm{a}}$ & $5.28^{\mathrm{a}}$ & $7.18^{\mathrm{b}}$ & $7.35^{\mathrm{b}}$ & 0.16 \\
Simpson & 15 & $0.90^{\mathrm{a}}$ & $0.93^{\mathrm{ab}}$ & $0.92^{\mathrm{a}}$ & $0.97^{\mathrm{b}}$ & $0.97^{\mathrm{b}}$ & 0.01 \\
OTUs & 15 & $982.9^{\mathrm{a}}$ & $1116^{\mathrm{a}}$ & $1325.0^{\mathrm{a}}$ & $2363.5^{\mathrm{b}}$ & $2664.7^{\mathrm{b}}$ & 94.6 \\
\hline
\end{tabular}

a,b,c Means within a row not bearing a common superscript differ $(p<0.05) .{ }^{1}$ CON $=$ No SmartCare or XPC; SCFP1 $=1 \mathrm{~g} /$ head $/ \mathrm{d}$ SmartCare in milk $+0.5 \%$ XPC in the starter grains; SCFP2 $=1 \mathrm{~g} / \mathrm{head} / \mathrm{d}$ SmartCare in milk $+1 \%$ $\mathrm{XPC}$ in the starter grains (Diamond V, Cedar Rapids, Iowa); Total means CON+SCFP1+SCPF2. ${ }^{2} \mathrm{~N}=$ the number of samples was used in each site. ${ }^{3}$ Mean =least square mean, RL56 = Rumen liquid portions at day 56, RS56 = Rumen solid contents at day 56, DC56= Duodenal contents at day 56, CC $=$ Caecal contents at day 56, RC $=$ Rectal contents at day 56. 
(a) ACE changes along the GIT

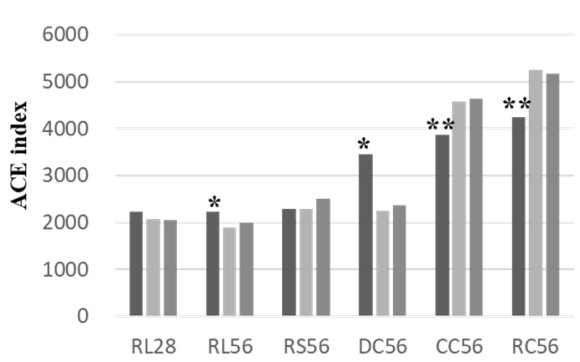

(c) Shannon changes along the GIT

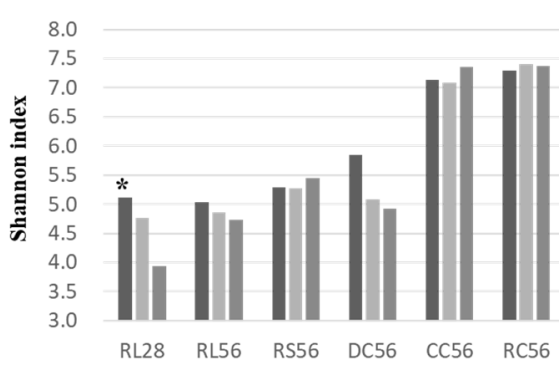

\section{(b) OTUs changes along the GIT}

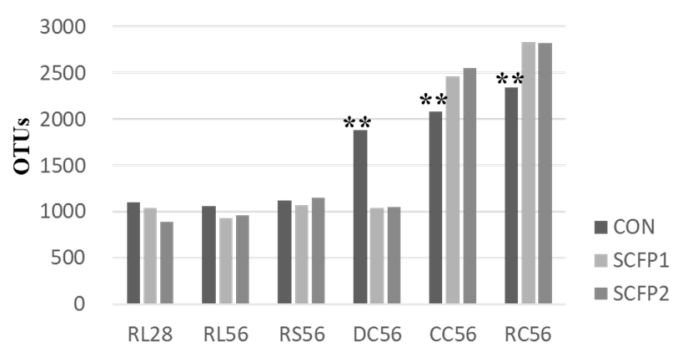

(d) Simpson changes along the GIT

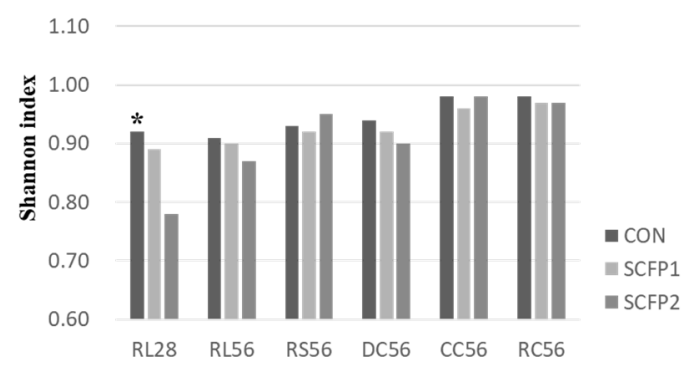

Figure 1. The difference of average (a) ACE, (b) OTU numbers, (c) Shannon and (d) Simpson indices values of CON, SCFP1 and SCFP2 along the GIT. CON $=$ No SmartCare and XPC; SCFP1 $=1 \mathrm{~g} / \mathrm{head} / \mathrm{d}$ SmartCare in milk $+0.5 \%$ XPC in the starter grains; SCFP2 $=1 \mathrm{~g} / \mathrm{head} / \mathrm{d}$ SmartCare in milk $+1 \%$ XPC in the starter grains (Diamond V, Cedar Rapids, Iowa). ${ }^{*} p<0.1,{ }^{* *} p<0.05$ between CON and SCFP (SCFP1 \& SCFP2). RL28 = Rumen liquid portions at day $28(n=13)$, RL56 = Rumen liquid portions at day $56(n=15)$, RS56 = Rumen solid contents at day $56(n=15)$, DC56= Duodenal contents at day 56 $(\mathrm{n}=15), C \mathrm{C}=$ Cecal contents at day $56(\mathrm{n}=15), \mathrm{RC}=$ Rectal contents at day $56(\mathrm{n}=15)$.

\subsubsection{Sample Clustering}

The Venn diagrams demonstrated inconsistent overlapping patterns for each treatment in different sites (Figure 2). Along the GIT, there were different numbers of unique OTUs among treatments in RL28 (CON, 728; SCFP1, 629; and SCFP2, 445), RL56 (CON, 507; SCFP1, 316; and SCFP2, 385), DC56 (CON, 1856; SCFP1, 373; and SCFP2, 359), in CC56 (CON, 619; SCFP1, 1033; and SCFP2, 1087) and RC56 (CON, 801; SCFP1, 1151; and SCFP2, 1196). Moreover, compared to shared OTUs between CON and SCFP in RL28 (180, accounting for $6.1 \%$ of detected OTUs), CC56 (341, accounting for 5.9\% of detected OTUs) and RC56 (341, accounting for 5.3\% of detected OTUs), greater OTU overlap between SCFP1 and SCFP2 was exhibited in RL28 (243, accounting for $8.2 \%$ of detected OTUs), CC56 (606, accounting for $10.5 \%$ of detected OTUs), and RC56 (705, accounting for $11.0 \%$ of detected OTUs), respectively.

Principal coordinate analysis (PCOA) plots clustered all samples mainly by location (Figure 3a and Figure S1, ANOSIM $p=0.001$ ). Then by treatment at RL 28 (Figure $3 \mathrm{c}$ and Figure S1, ANOSIM $p=0.03$ ) and RC56 (Figure 3d and Figure S1, ANOSIM $p=0.10$ ). We did not observe strong clustering among treatments variables in other sites (Figure 3b, Figures S2 and S3, ANOSIM $p>0.05$ ), suggesting that with the exception of location, supplementing SCFP has the potential to change the microbial community in early age rumen and in the rectum of the weaning calves. 


\section{RL28}

RL56

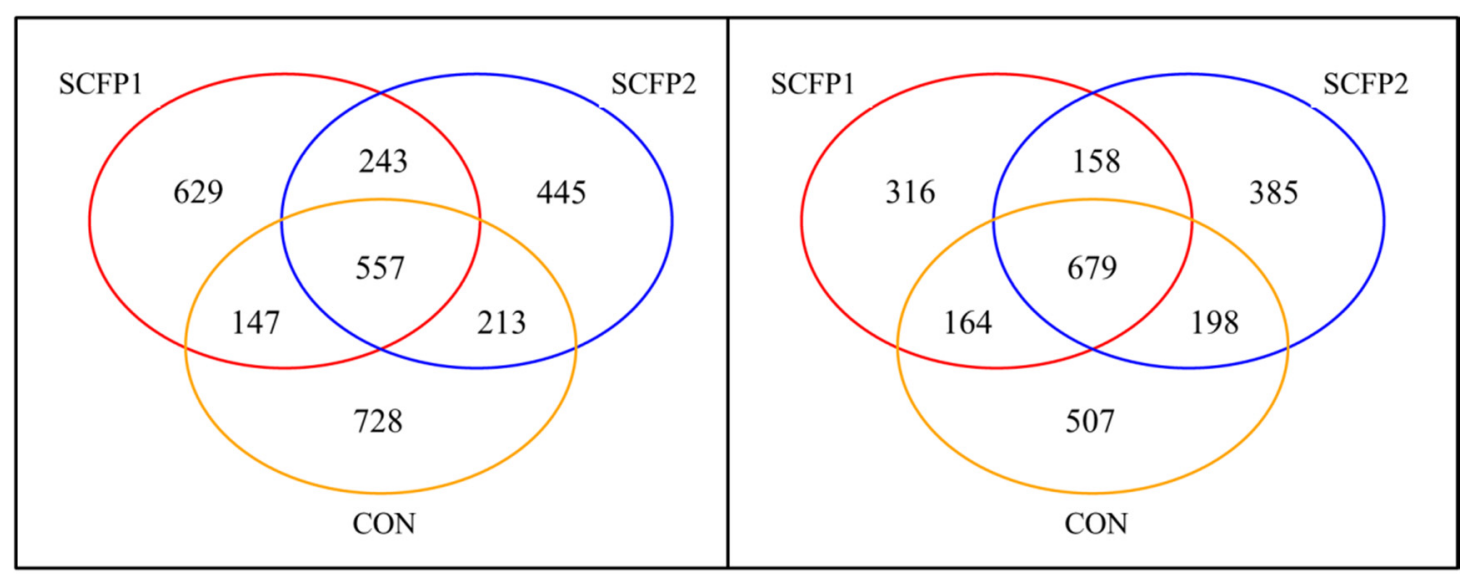

\section{$\begin{array}{ll}\text { RS56 DC56 } & \text { D5 }\end{array}$}

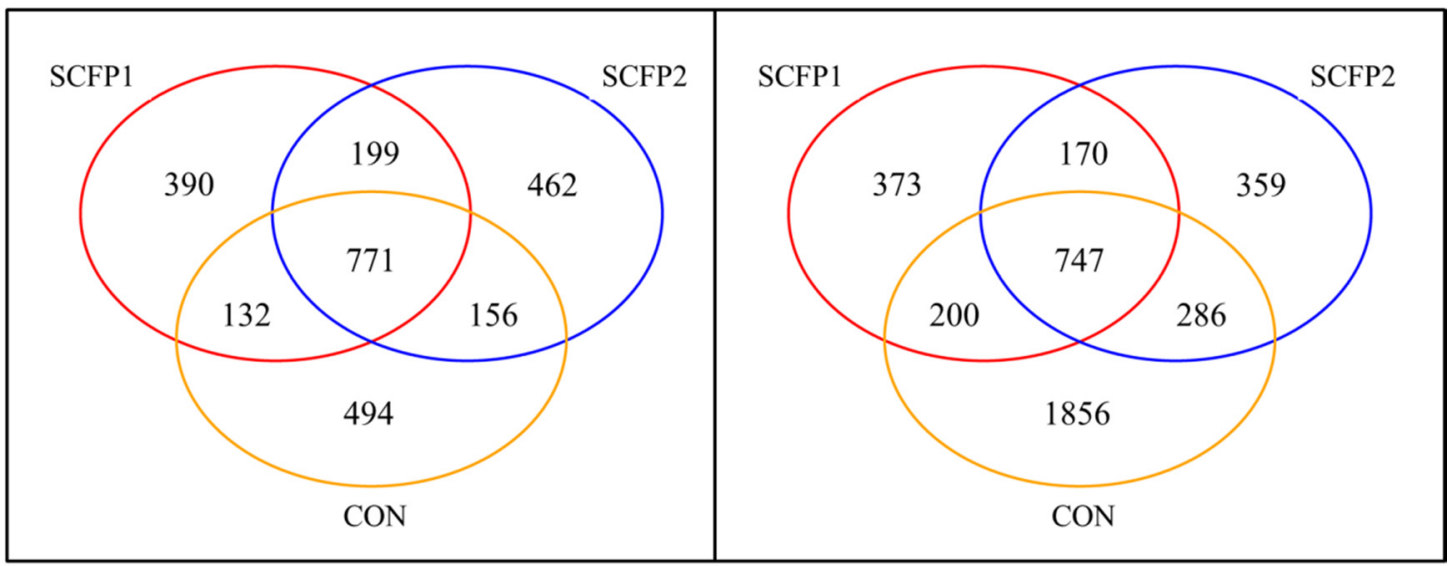

\section{$\begin{array}{ll}\text { CC56 RC56 } & \end{array}$}

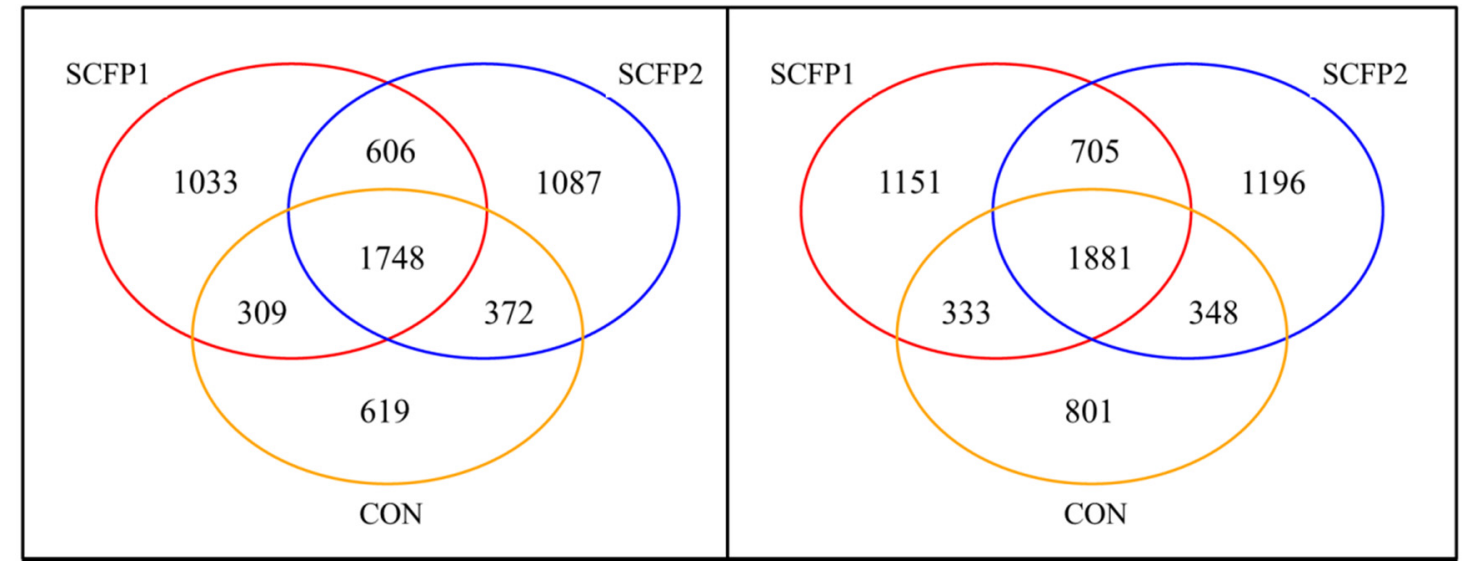

Figure 2. Venn plot for OTUs. The OTUs difference among CON, SCFP1 and SCFP2 in digestive sites respectively (RL28, RL56, RS56, DC56, CC56 and RC56). Singleton sequences (occurring once in only one sample) were removed before analysis. 
a

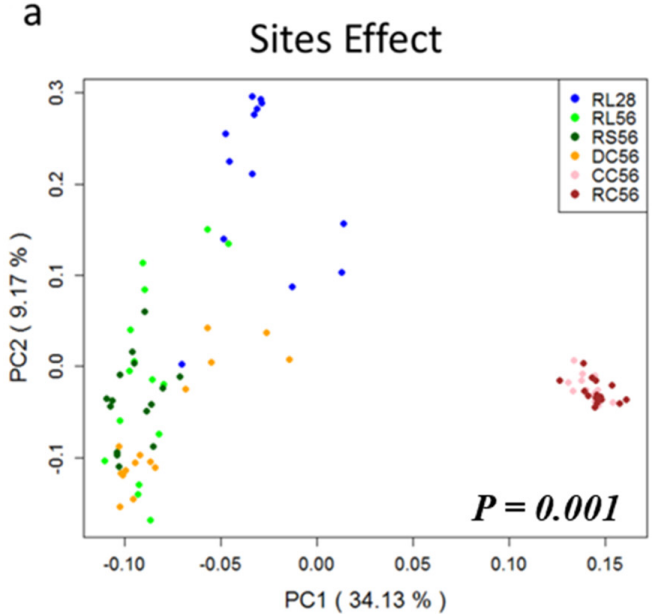

C

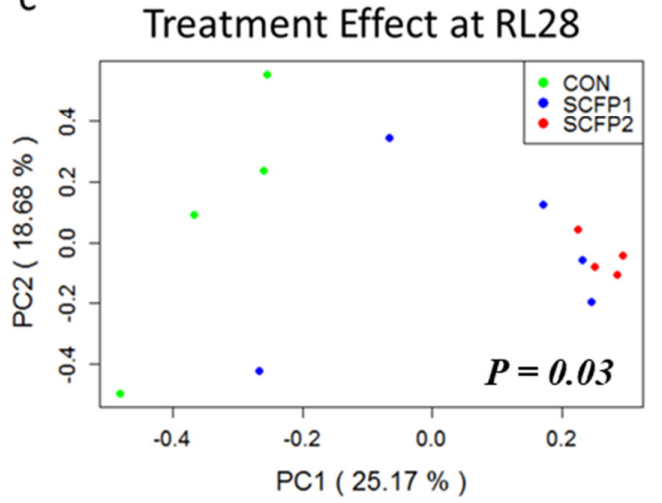

b

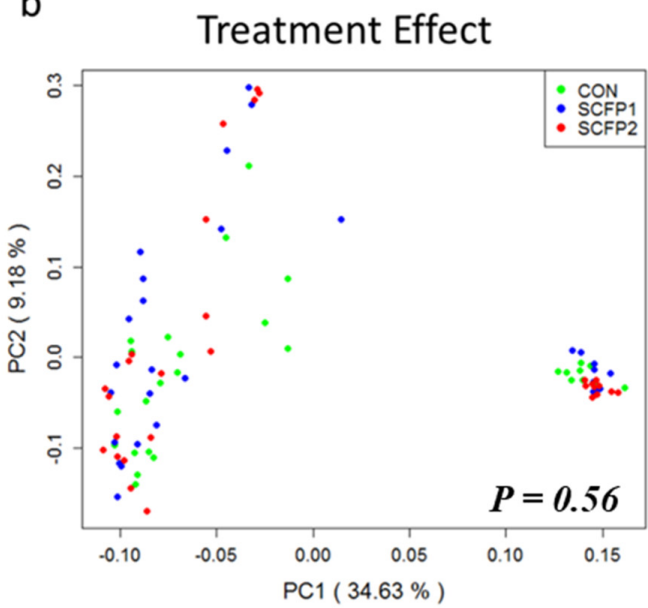

d

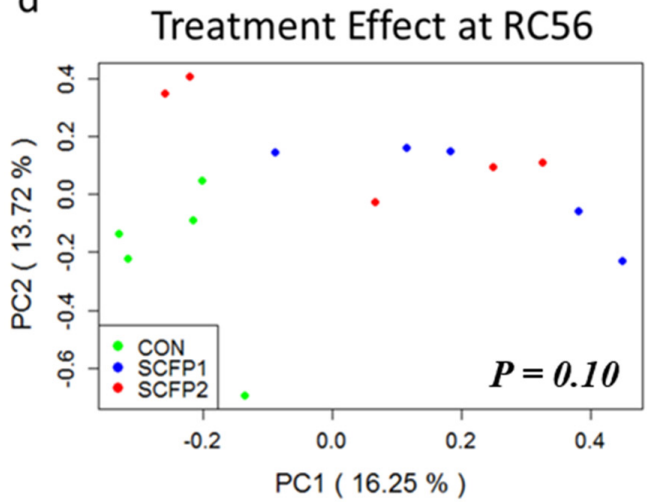

Figure 3. Separation of samples based on phylogenetic information by using PCoA plot, statistical comparison of microbiota was performed with ANOSIM analysis (a), sample clustering into variations by locations. (b), Sample clustering into variations by treatments. (c), Treatment separation in RL28. (d), Treatment separation in RC56.

\subsubsection{Taxonomic Characteristics}

Overall, 25, 21 and 20 phyla and 256, 247 and 229 genera were detected for CON, SCFP1 and SCFP2, respectively. In RL28 (Table S1), seven predominant phyla (Firmicutes, Bacteroidetes, Actinobacteria, Proteobacteria, Tenericutes, Cyanobacteria and Spirochaetes with at least $\geq 1 \%$ relative abundance in one sample) were detected. Among these phyla, SCFP supplementation decreased relative occurrence of Bacteroidetes $(p<0.001)$ and Spirochaetes $(p=0.04)$, and increased that of Firmicutes $(p=0.02)$. The impact of SCFP on core family Prevotellaceae $(p=0.002)$ and Lachnospiraceae $(p=0.06)$ significantly contributed to the lower abundance of Bacteroidetes $(p<0.001)$ and higher abundance of Firmicutes ( $p=0.02$ ), respectively (Figure 4 ). On the other hand, genus Prevotella and Butyrivibrio were dominant in Prevotellaceae and Lachnospiraceae, respectively. The relatively low abundance genus, Mogibacterium $(p=0.06)$ increased while Sphaerochaeta $(p=0.04)$ decreased when supplementing SCFP in milk and starter.

In rumen and duodenum samples at day 56 , almost no significant treatment effects were observed in the RL56, RS56 and DC56 at family (Figure 4) or any other level of classification (Tables S2-S4, $p>0.05)$. However, in large intestine samples at day 56, relative occurrence of family Lachnospiraceae were found to decrease in RC56 (CON, 13.09\%; SCFP1, 9.14\%; and SCFP2, 9.31\%, $p=0.04$ ). Numerical increase was exhibited in family Ruminococcus in RC56 (CON, 26.05\%; SCFP1, 38.15\%; and SCFP2, $33.48 \%, p=0.14$ ) due to the supplementation of SCFP (Figure 4). The increase in Ruminococcus was likely due to genus the Ruminococcus and Oscillospira (Tables S5 and S6). 
No significant effects were observed between SCFP1 and SCFP2 for bacteria abundance in all GIT sites at day 56.

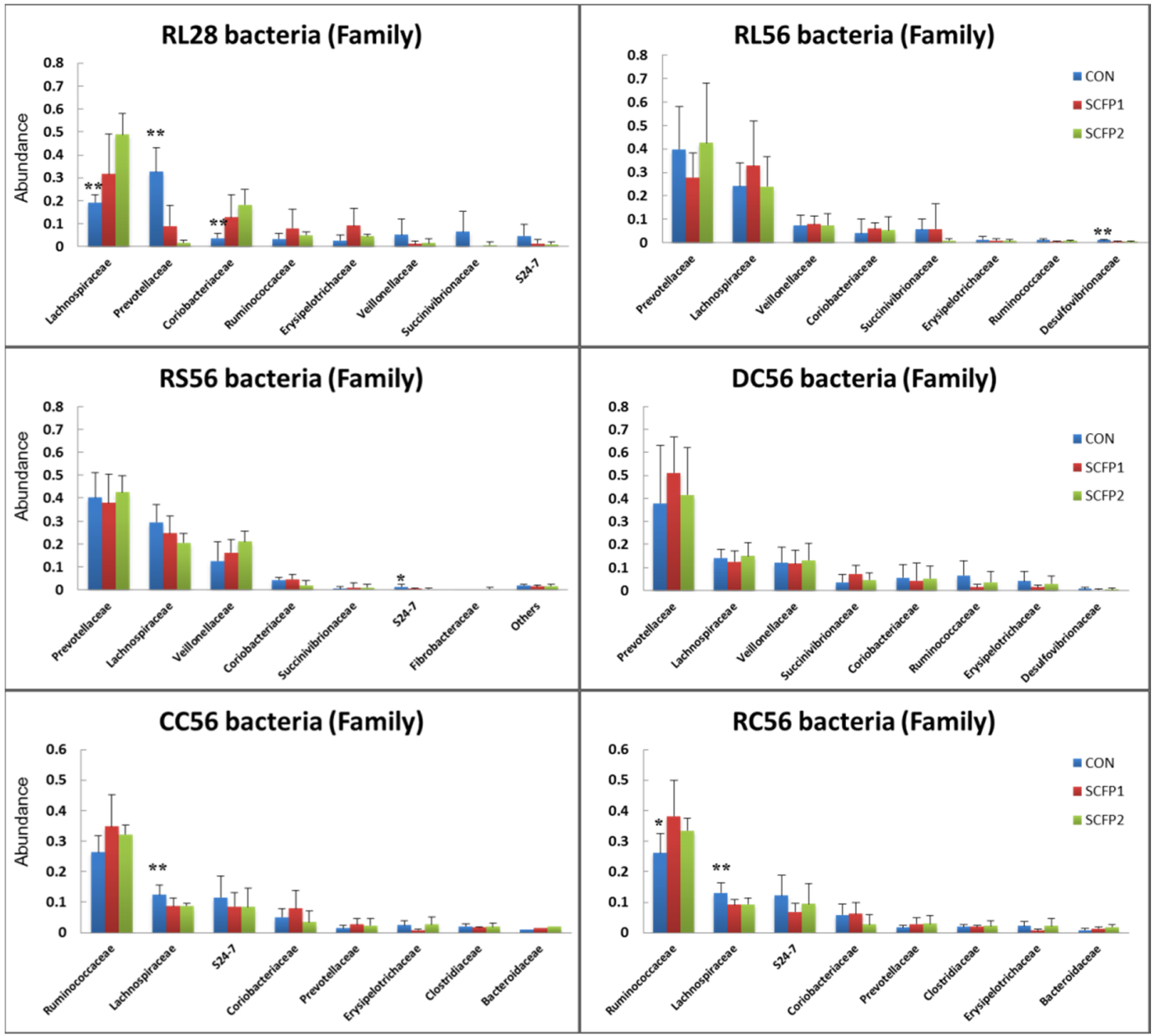

Figure 4. Top eight bacteria percentage in family level of total OTUs number of ( $\square$ ) CON, ( $\square$ ) SCFP1 and $(\square)$ SCFP2 through GITs. CON = No SmartCare and XPC; SCFP1 = $1 \mathrm{~g} / \mathrm{head} / \mathrm{d}$ SmartCare in milk $+0.5 \%$ XPC in the starter grains; SCFP2 $=1 \mathrm{~g} / \mathrm{head} / \mathrm{d}$ SmartCare in milk $+1 \%$ XPC in the starter grains (Diamond V, Cedar Rapids, Iowa). ${ }^{*} p<0.1,{ }^{* *} p<0.05$ between CON and SCFP (SCFP1 \& SCFP2). RL28 = Rumen liquid portions at day $28(n=13)$, RL56 = Rumen liquid portions at day $56(n=15)$, RS56 = Rumen solid contents at day $56(n=15)$, DC56 = Duodenal contents at day $56(n=15), C C=$ Cecal contents at day $56(n=15), R C=$ Rectal contents at day $56(n=15)$.

\section{Discussion}

According to our results based on microbial alpha-diversity (Figure 1), SCFP can reduce microbial richness and evenness in rumen and increase community richness in large intestine. In other words, with supplementation of SCFP, less of different types of bacteria were detected in rumen but more types in the large intestine. A decrease in rumen community richness in SCFP groups was probably the result of the higher emergence of dominant bacteria in SCFP (Figure 4), which affected the colonization by other bacteria. The improvement in the core bacteria might change the rumen environment and as a result affect the emergence of other bacteria. For example, the Lachnospiraceae, butyrate-producing bacteria $[28,29]$, increased in RL28 in SCFP (CON vs. SCFP $=19.21 \%$ vs. $40.25 \%$, Figure 4 ), which concomitantly resulted in a higher butyrate concentration [18]. Li, et al. [30] demonstrated that butyrate infusion altered rumen microbial composition and lowered the numbers of OTUs. Hence, we speculate that the decrease in community richness and diversity in current study was probably induced by the 
increase in abundance of butyrate-producing bacteria (Lachnospiraceae) and the butyrate concentration in the rumen.

Saccharomyces cerevisiae fermentation products are a rich in nutritional metabolites, mannan oligosaccharides and $\beta$-glucans, which can benefit various types of bacteria [15]. In a companion study, no treatment differences were observed for starter intake [18], hence we postulate that the effects hereby observed came from SCFP rather than the ration. In agreement with findings in the large intestine, previous studies revealed that inclusion SCFP considerably stimulated the microbial richness in pigs [31], which might be associated with the SCFP metabolites ability to stimulate diverse communities of microorganisms that colonize the large intestine [32]. Although microbial richness increased in current study, the impact of SCFP on microbial richness and diversity has not been studied extensively, hence future research should aim at verifying the changes observed in the present study.

It is generally believed that SCFP can modulate the structure of bacterial community in a mature rumen $[33,34]$ and the large intestine in non-ruminants [32,35]. Similar impacts were obtained in the GIT of calves in this study (Figures 1 and 4). Greater effect of SCFP was found in rumen at an early age (day 28, Figure 3) when microbiota was less stable and more heterogeneous compared to more mature age [2]. Another reason might have been related to the Smartcare feeding from day 2 to day 30, whereby both XPC and Smartcare had an effect on rumen fermentation at day 28. At day 56, similar bacterial community was seen in the rumen when only XPC were added in the starter only (Figure 3). Although no big differences existed in the rumen at day 56, we found SCFP could affect rectal microbial community drastically (Figure 3, Table S6) which could influence fiber fermentation in the hindgut of calves. As young calves have a nonfunctional rumen, it is expected for SCFP to have greater impact on the hindgut rather than the rumen. These results suggest that both rumen and hindgut should be explored when investigating the effect of SCFP or other feed additives on calves in the future.

In line with previous bovine studies [36,37], the members of the Prevotellaceae, Lachnospiraceae, Coriobacteriaceae, and Ruminococcaceae were predominant in current study (Figure 4). This is the first time that we have demonstrated that these groups of bacteria are affected by SCFP in young calves. Coriobacteriaceae, belongs to pylum Actinobacteria, gram-positive bacteria that have been isolated from large intestine in both human and mouse, which was trended increased in SCFP groups $(p=0.08)$. They are associated with polyphenol conversion as well as bile acid and hepatic lipid metabolism [38]. In cattle, they represent up to $3 \%$ of mature rumen bacteria [39], which was similar to the current study. However, their role and function in rumen is largely unknown and need further investigation to explain the presence of higher Coriobacteriaceae in the present study when SCFP was supplemented.

Prevotellaceae, belongs to phylum Bacteroidetes, gram-negative bacteria with the ability to utilize various sugars [40] and are believed to play a crucial role in starch degradation [41]. Members of the Ruminococcaceae and Lachnospiraceae families are largely fibrolytic bacteria and belong to the phylum Firmicutes [36]. The lower emergence of Prevotellaceae and higher emergence of Lachnospiraceae suggest that SCFP might change the fermentation type and stimulate the degradation of recalcitrant fiber substrates in pre-mature rumen of calves.

In the large intestine, as the essential types of bacteria that produce SCFAs, Lachnospiraceae and Ruminococcaceae are significantly depleted in diarrheic patients [42]. The abundance of Ruminococcaceae increased (CON vs. SCFP $=26.23 \%$ vs. $34.66 \%$ ) while that of Lachnospiraceae decreased (CON vs. $\mathrm{SCFP}=12.72 \%$ vs. $8.95 \%$ ) in SCFP groups compared to CON. A recent comparitive genomic study on the carbohydrate-active enzymes, transporters and metabolic pathways between Lachnospiraceae and Ruminococcaceae, revealed that although these two members were specialized in the degradation of plant material, Lachnospiraceae were richer in starch-degrading alpha-glucosidases and phosphorylases genes while Ruminococcaceae were rich in cellulase genes and endo-1, 4-beta-xylanases [43]. Therefore, we speculate that the higher abundance of Ruminococcaceae in SCFP groups in CC56 and RC56 might stimulate the degradation of cellulose, which has not or been poorly digested in the rumen with less than $1 \%$ of Ruminococcaceae in each group (Table S2). On the other hand, the lower Lachnospiraceae in SCFP groups in large intestine might be because of the higher Lachnospiraceae (dominated by genus 
Butyrivibrio) exhibited in rumen, where most of the substrates for Lachnospiraceae had already been largely utilized, resulting in less residues arriving in large intestine when SCFP was supplemented. In a companion paper [18], we found SCFP groups exhibited a higher butyrate production in the rumen. The higher butyrate was probably produced by butyrate-producing anaerobic bacteria, genus Butyrivibrio [28], which is further proof that specific substrates were utilized by higher Lachnospiraceae in SCFP groups in rumen. Although our data clearly showed differences in the microbiota structure as a result of dietary supplementation with SCFP, deciphering these changes at the family or other levels and relating these changes to ecological function remain a formidable challenge and need further investigation.

\section{Conclusions}

This study demonstrated that the bacterial composition of pre-weaned calves varied by the dietary supplementation of SCFP. Saccharomyces cerevisiae fermentation products have an ability to increase the species richness in large intestine. It also changed the bacterial composition and stimulated the fiber digesting bacteria in rumen at early age (RL28) and later in large intestine (RC56). Furthermore, SCFP are more likely to change the bacterial composition in hindgut rather than rumen at day 56, as SCFP supplemented calves increased the abundance Ruminococcaceae in large intestine. When calves were supplemented with SCFP at a higher rate in the starter ( $1 \%$ vs. $0.5 \%$ ) no further changes were observed in bacterial community.

Supplementary Materials: The following are available online at http:/ /www.mdpi.com/2076-2615/9/1/4/s1, Figure S1: ANOSIM analysis were performed at sites (a) and treatments in GITs (b), RL28. (c), and RC56 (d), Figure S2: ANOSIM analysis were performed among treatments in RL56 (a), RS56 (b), DC56 (c) and CC56 (d), Figure S3: Separation of samples based on phylogenetic information using PCoA plot. (a), treatment separation in RL56. (b), treatment separation in RS56. (c), treatment separation in DC56. (d), treatment separation in CC56, Table S1: The effect of SCFP on Bacterial abundance in each level in rumen liquid fraction sampled on day 28 (RL28), Table S2: The effect of SCFP on Bacterial abundance in each level in rumen liquid fraction sampled on day 56 (RL56), Table S3: The effect of SCFP on Bacterial abundance in each level in rumen solid fraction sampled on day 56 (RS56), Table S4: The effect of SCFP on Bacterial abundance in each level in duodenal content sampled on day 56 (DC56), Table S5: The effect of SCFP on Bacterial abundance in each level in cecal content sampled on day 56 (CC56); Table S6: The effect of SCFP on Bacterial abundance in each level in rectal content sampled on day 56 (RC56).

Author Contributions: Conceptualization, Z.C. and S.L.; methodology, J.X. and I.Y.; software, J.X. and S.J.; formal analysis, J.X.; resources, S.L., I.Y. and R.C.; data curation, J.X. and S.D.; writing-original draft preparation, J.X.; writing-review and editing, Z.C. and G.M.A.; supervision, Z.W. and R.C.; project administration, J.X. and G.M.A.; funding acquisition, Z.C.

Funding: This work was supported by Diamond V Mills Inc. (No. DVIP 147121, Cedar Rapids, IA) and Tianjin Commission of Science Technology (15YFXQNC00020, Tianjin, China).

Acknowledgments: We thank the Dairy Teaching and Research Farm (Shunyi, Beijing, China) for use of their animals and facilities.

\section{References}

1. Rey, M.; Enjalbert, F.; Combes, S.; Cauquil, L.; Bouchez, O.; Monteils, V. Establishment of ruminal bacterial community in dairy calves from birth to weaning is sequential. J. Appl. Microbiol. 2014, 116, $245-257$. [CrossRef] [PubMed]

2. Jami, E.; Israel, A.; Kotser, A.; Mizrahi, I. Exploring the bovine rumen bacterial community from birth to adulthood. ISME J. 2013, 7, 1069-1079. [CrossRef] [PubMed]

3. Smith, H.W. The development of the flora of the alimentary tract in young animals. J. Pathol. Bacteriol. 1965, 90, 495-513. [CrossRef] [PubMed]

4. Vlková, E.; Trojanová, I.; Rada, V. Distribution of bifidobacteria in the gastrointestinal tract of calves. Folia Microbiol. 2006, 51, 325-328. [CrossRef]

5. Guzman, C.E.; Bereza-Malcolm, L.T.; De, G.B.; Franks, A.E. Presence of Selected Methanogens, Fibrolytic Bacteria, and Proteobacteria in the Gastrointestinal Tract of Neonatal Dairy Calves from Birth to 72 Hours. PLoS ONE 2015, 10, e0133048. [CrossRef] [PubMed] 
6. Kleinjöbstl, D.; Schornsteiner, E.; Mann, E.; Wagner, M.; Drillich, M.; Schmitzesser, S. Pyrosequencing reveals diverse fecal microbiota in Simmental calves during early development. Front. Microbiol. 2014, 5, 622.

7. Castro, J.J.; Gomez, A.; White, B.; Loften, J.R.; Drackley, J.K. Changes in the intestinal bacterial community, short-chain fatty acid profile, and intestinal development of preweaned Holstein calves. 2. Effects of gastrointestinal site and age. J. Dairy Sci. 2016, 99, 9703-9715. [CrossRef]

8. Dawson, K.; Newman, K.; Boling, J. Effects of microbial supplements containing yeast and lactobacilli on roughage-fed ruminal microbial activities. J. Anim. Sci. 1990, 68, 3392-3398. [CrossRef]

9. Chaucheyras-Durand, F.; Fonty, G. Establishment of cellulolytic bacteria and development of fermentative activities in the rumen of gnotobiotically-reared lambs receiving the microbial additive Saccharomyces cerevisiae CNCM I-1077. Reprod. Nutr. Dev. 2001, 41, 57-68. [CrossRef]

10. Hristov, A.N.; Varga, G.; Cassidy, T.; Long, M.; Heyler, K.; Karnati, S.K.R.; Corl, B.; Hovde, C.J.; Yoon, I. Effect of Saccharomyces cerevisiae fermentation product on ruminal fermentation and nutrient utilization in dairy cows. J. Dairy Sci. 2010, 93, 682-692. [CrossRef]

11. Magalhães, V.J.; Susca, F.; Lima, F.S.; Branco, A.F.; Yoon, I.; Santos, J.E. Effect of feeding yeast culture on performance, health, and immunocompetence of dairy calves. J. Dairy Sci. 2008, 91, 1497-1509. [CrossRef] [PubMed]

12. Lesmeister, K.E.; Heinrichs, A.J.; Gabler, M.T. Effects of supplemental yeast (Saccharomyces cerevisiae) culture on rumen development, growth characteristics, and blood parameters in neonatal dairy calves. J. Dairy Sci. 2004, 87, 1832-1839. [CrossRef]

13. Quigley, J.D.; Boehms, S.I.; Steen, T.M.; Heitmann, R.N. Effects of lasalocid on selected ruminal and blood metabolites in young calves. J. Dairy Sci. 1992, 75, 2235-2241. [CrossRef]

14. Brewer, M.T.; Anderson, K.L.; Yoon, I.; Scott, M.F.; Carlson, S.A. Amelioration of salmonellosis in pre-weaned dairy calves fed Saccharomyces cerevisiae fermentation products in feed and milk replacer. Vet. Microbiol. 2014, 172, 248-255. [CrossRef] [PubMed]

15. Callaway, E.S.; Martin, S.A. Effects of a Saccharomyces cerevisiae Culture on Ruminal Bacteria that Utilize Lactate and Digest Cellulose. J. Dairy Sci. 1997, 80, 2035-2044. [CrossRef]

16. He, S.; Zhou, Z.; Meng, K.; Zhao, H.; Yao, B.; Ringø, E.; Yoon, I. Effects of dietary antibiotic growth promoter and Saccharomyces cerevisiae fermentation product on production, intestinal bacterial community, and nonspecific immunity of hybrid tilapia (Oreochromis niloticus female $x$ Oreochromis aureus male). J. Anim. Sci. 2011, 89, 84-92. [CrossRef]

17. Zhu, W.; Wei, Z.; Xu, N.; Yang, F.; Yoon, I.; Chung, Y.; Liu, J.; Wang, J. Effects of Saccharomyces cerevisiae fermentation products on performance and rumen fermentation and microbiota in dairy cows fed a diet containing low quality forage. J. Anim. Sci. Biotechnol. 2017, 8, 677-685. [CrossRef]

18. Xiao, J.; Alugongo, G.; Chung, R.; Dong, S.; Li, S.; Yoon, I.; Wu, Z.; Cao, Z. Effects of Saccharomyces cerevisiae fermentation products on dairy calves: Ruminal fermentation, gastrointestinal morphology, and microbial community. J. Dairy Sci. 2016. [CrossRef]

19. NRC. Nutrient Requirements of Dairy Cattle, 7th ed.; NRC: Rockville, MD, USA, 2001; p. 381.

20. Caporaso, J.G.; Lauber, C.L.; Walters, W.A.; Berglyons, D.; Huntley, J.; Fierer, N.; Owens, S.M.; Betley, J.; Fraser, L.; Bauer, M. Ultra-high-throughput microbial community analysis on the Illumina HiSeq and MiSeq platforms. ISME J. Multidiscip. J. Microb. Ecol. 2012, 6, 1621-1624. [CrossRef]

21. Magoč, T.; Salzberg, S.L. FLASH: Fast length adjustment of short reads to improve genome assemblies. Bioinformatics 2011, 27, 2957-2963. [CrossRef]

22. Caporaso, J.G.; Kuczynski, J.; Stombaugh, J.; Bittinger, K.; Bushman, F.D.; Costello, E.K.; Fierer, N.; Pena, A.G.; Goodrich, J.K.; Gordon, J.I. QIIME allows analysis of high-throughput community sequencing data. Nat. Methods 2010, 7, 335-336. [CrossRef] [PubMed]

23. DeSantis, T.Z.; Hugenholtz, P.; Larsen, N.; Rojas, M.; Brodie, E.L.; Keller, K.; Huber, T.; Dalevi, D.; Hu, P.; Andersen, G.L. Greengenes, a chimera-checked $16 \mathrm{~S}$ rRNA gene database and workbench compatible with ARB. Appl. Environ. Microbiol. 2006, 72, 5069-5072. [CrossRef] [PubMed]

24. Wang, Q.; Garrity, G.M.; Tiedje, J.M.; Cole, J.R. Naive Bayesian classifier for rapid assignment of rRNA sequences into the new bacterial taxonomy. Appl. Environ. Microbiol. 2007, 73, 5261-5267. [CrossRef] [PubMed] 
25. Dillies, M.-A.; Rau, A.; Aubert, J.; Hennequet-Antier, C.; Jeanmougin, M.; Servant, N.; Keime, C.; Marot, G.; Castel, D.; Estelle, J. A comprehensive evaluation of normalization methods for Illumina high-throughput RNA sequencing data analysis. Brief. Bioinform. 2013, 14, 671-683. [CrossRef] [PubMed]

26. Kolde, R. Pheatmap: Pretty heatmaps. In R Package Version 2.4-1; Microsoft: Redmond, WA, USA, 2012.

27. Stearns, J.C.; Lynch, M.D.; Senadheera, D.B.; Tenenbaum, H.C.; Goldberg, M.B.; Cvitkovitch, D.G.; Croitoru, K.; Moreno-Hagelsieb, G.; Neufeld, J.D. Bacterial biogeography of the human digestive tract. Sci. Rep. 2011, 1, 170. [CrossRef] [PubMed]

28. Mrazek, J.; Tepšič, K.; Avguštin, G.; Kopečný, J. Diet-dependent shifts in ruminal butyrate-producing bacteria. Folia Microbiol. 2006, 51, 294-298. [CrossRef]

29. Meehan, C.J.; Beiko, R.G. A phylogenomic view of ecological specialization in the Lachnospiraceae, a family of digestive tract-associated bacteria. Genome Boil. Evol. 2014, 6, 703-713. [CrossRef]

30. Li, R.W.; Wu, S.; Vi, R.L.B.; Li, W.; Li, C. Perturbation dynamics of the rumen microbiota in response to exogenous butyrate. PLoS ONE 2012, 7, e29392. [CrossRef]

31. Kiarie, E.; Bhandari, S.; Scott, M.; Krause, D.; Nyachoti, C. Growth performance and gastrointestinal microbial ecology responses of piglets receiving Saccharomyces cerevisiae fermentation products after an oral challenge with Escherichia coli (K88). J. Anim. Sci. 2011, 89, 1062-1078. [CrossRef]

32. Price, K.; Totty, H.; Lee, H.; Utt, M.; Fitzner, G.; Yoon, I.; Ponder, M.; Escobar, J. Use of fermentation product on growth performance and microbiota of weaned pigs during infection. J. Anim. Sci. 2010, 88, 3896-3908. [CrossRef]

33. Harrison, G.; Hemken, R.; Dawson, K.; Harmon, R.; Barker, K. Influence of addition of yeast culture supplement to diets of lactating cows on ruminal fermentation and microbial populations. J. Dairy Sci. 1988, 71, 2967-2975. [CrossRef]

34. Mullins, C.; Mamedova, L.; Carpenter, A.; Ying, Y.; Allen, M.; Yoon, I.; Bradford, B. Analysis of rumen microbial populations in lactating dairy cattle fed diets varying in carbohydrate profiles and Saccharomyces cerevisiae fermentation product. J. Dairy Sci. 2013, 96, 5872-5881. [CrossRef] [PubMed]

35. He, S.; Zhou, Z.; Liu, Y.; Shi, P.; Yao, B.; Ringø, E.; Yoon, I. Effects of dietary Saccharomyces cerevisiae fermentation product (DVAQUA ${ }^{\circledR}$ ) on growth performance, intestinal autochthonous bacterial community and non-specific immunity of hybrid tilapia (Oreochromis niloticus $+\times$ O. aureus $\sigma^{7}$ ) cultured in cages. Aquaculture 2009, 294, 99-107. [CrossRef]

36. Thoetkiattikul, H.; Mhuantong, W.; Laothanachareon, T.; Tangphatsornruang, S.; Pattarajinda, V.; Eurwilaichitr, L.; Champreda, V. Comparative analysis of microbial profiles in cow rumen fed with different dietary fiber by tagged 16S rRNA gene pyrosequencing. Curr. Microbiol. 2013, 67, 130-137. [CrossRef] [PubMed]

37. Golder, H.; Denman, S.; McSweeney, C.; Celi, P.; Lean, I. Ruminal bacterial community shifts in grain-, sugar-, and histidine-challenged dairy heifers. J. Dairy Sci. 2014, 97, 5131-5150. [CrossRef] [PubMed]

38. Clavel, T.; Lepage, P.; Charrier, C. The Family Coriobacteriaceae. In The Prokaryotes; Springer: New York, NY, USA, 2014; pp. 201-238.

39. Šulák, M.; Sikorová, L.; Jankuvová, J.; Javorský, P.; Pristaš, P. Variability of Actinobacteria, a minor component of rumen microflora. Folia Microbiol. 2012, 57, 351-353. [CrossRef] [PubMed]

40. Morotomi, M.; Nagai, F.; Sakon, H.; Tanaka, R. Paraprevotella clara gen. nov., sp. nov. and Paraprevotella xylaniphila sp. nov., members of the family 'Prevotellaceae'isolated from human faeces. Int. J. Syst. Evol. Microbiol. 2009, 59, 1895-1900. [CrossRef]

41. Hobson, P.N.; Stewart, C.S. The Rumen Microbial Ecosystem; Springer Science \& Business Media: New York, NY, USA, 2012.

42. Antharam, V.C.; Li, E.C.; Ishmael, A.; Sharma, A.; Mai, V.; Rand, K.H.; Wang, G.P. Intestinal dysbiosis and depletion of butyrogenic bacteria in Clostridium difficile infection and nosocomial diarrhea. J. Clin. Microbiol. 2013, 51, 2884-2892. [CrossRef]

43. Biddle, A.; Stewart, L.; Blanchard, J.; Leschine, S. Untangling the genetic basis of fibrolytic specialization by Lachnospiraceae and Ruminococcaceae in diverse gut communities. Diversity 2013, 5, 627-640. [CrossRef]

(C) 2018 by the authors. Licensee MDPI, Basel, Switzerland. This article is an open access article distributed under the terms and conditions of the Creative Commons Attribution (CC BY) license (http:/ / creativecommons.org/licenses/by/4.0/). 